This item was submitted to Loughborough's Research Repository by the author.

Items in Figshare are protected by copyright, with all rights reserved, unless otherwise indicated.

\title{
Preparation, characterization and properties of polycaprolactone diol- functionalized multi-walled carbon nanotube/thermoplastic polyurethane composite
}

\section{PLEASE CITE THE PUBLISHED VERSION}

http://dx.doi.org/10.1016/j.compositesa.2014.10.028

\section{PUBLISHER}

(C) Elsevier Ltd.

\section{VERSION}

AM (Accepted Manuscript)

\section{PUBLISHER STATEMENT}

This work is made available according to the conditions of the Creative Commons Attribution-NonCommercialNoDerivatives 4.0 International (CC BY-NC-ND 4.0) licence. Full details of this licence are available at: https://creativecommons.org/licenses/by-nc-nd/4.0/

\section{LICENCE}

CC BY-NC-ND 4.0

\section{REPOSITORY RECORD}

Jing, Qifei, Jia Yan Law, Lay Poh Tan, Vadim V. Silberschmidt, Lin Li, and ZhiLi Dong. 2019. "Preparation, Characterization and Properties of Polycaprolactone Diol-functionalized Multi-walled Carbon Nanotube/thermoplastic Polyurethane Composite". figshare. https://hdl.handle.net/2134/17468. 


\title{
Preparation, characterization and properties of polycaprolactone diol- functionalized multi-walled carbon nanotube/thermoplastic polyurethane composite
}

\author{
Qifei Jing ${ }^{\text {a,b,d }}$, Jia Yan Law ${ }^{\text {a,c }}$, Lay Poh Tan ${ }^{\text {a }}$, Vadim V. Silberschmidt ${ }^{\text {d }}$, Lin Li ${ }^{\text {e, }}$, ZhiLi Dong ${ }^{\text {a,b, }}$ \\ ${ }^{a}$ School of Materials Science and Engineering, Nanyang Technological University, 50 Nanyang Avenue, Singapore 639798, Singapore \\ ${ }^{\mathrm{b}}$ Institute for Sports Research, Nanyang Technological University, 50 Nanyang Avenue, Singapore 639798, Singapore \\ ${ }^{c}$ Facility for Analysis, Characterization, Testing and Simulation, Nanyang Technological University, 50 Nanyang Avenue, Singapore 639798, Singapore \\ ${ }^{\mathrm{d}}$ Wolfson School of Mechanical and Manufacturing Engineering, Loughborough University, Loughborough, Leicestershire LE11 3TU, UK \\ ${ }^{\mathrm{e}}$ School of Mechanical and Aerospace Engineering, Nanyang Technological University, 50 Nanyang Avenue, Singapore 639798, Singapore
}

\begin{abstract}
Multi-walled carbon nanotubes (MWCNTs) were chemically functionalized to prepare thermoplastic polyurethane (PU) composites with enhanced properties. In order to achieve a high compatibility of functionalized MWCNTs with the PU matrix, polycaprolactone diol (PCL), as one of PU's monomers, was selectively grafted on the surface of MWCNTs (MWCNT-PCL), while carboxylic acid groups function- alized MWCNTs (MWCNT-COOH) and raw MWCNTs served as control. Both MWCNT-COOH and MWCNT-PCL improved the dispersion of MWCNTs in the PU matrix and interfacial bonding between them at $1 \mathrm{wt} \%$ loading fraction. The MWCNT-PCL/PU composite showed the greatest extent of improve- ment, where the tensile strength and modulus were $51.2 \%$ and $33.5 \%$ higher than those of pure PU respec- tively, without sacrificing the elongation at break. The considerable improvement in both mechanical properties and thermal stability of MWCNT-PCL/PU composite should result from the homogeneous dispersion of MWCNT-PCL in the PU matrix and strong interfacial bonding between them.
\end{abstract}

\section{Introduction}

Carbon nanotubes (CNTs) have received considerable attention because of their distinctive structures and fascinating properties since first reported in 1991 [1]. Researchers show great interests in utilizing CNTs in various areas, such as electrochemical biosensors [2,3], nanowires [4], nanoprobes for high-resolution imaging [5], electromechanical actuators [6], and other applications [7,8]. Among them, polymer-based CNTs composite has been a research focus for many years. Various polymer materials have been studied as the composite matrices, such as polypyrrole [9], poly(vinyl alcohol) [10], polystyrene [11], poly(methyl methacrylate) [12] and liquid crystalline polymers [13]. The excellent mechanical, electrical, and thermal properties of carbon nanotube [14-16], combined with its high aspect ratio, make it a very promising reinforcing filler.

\footnotetext{
$\Uparrow$ Corresponding authors at: N4.1-01-04, School of Materials Science and Engi-
} neering, Nanyang Technological University, 50 Nanyang Avenue, Singapore 639798, Singapore (Z. Dong). Tel.: +65 67906727.

E-mail address: zldong@ntu.edu.sg_(Z. Dong).
However, the application of CNTs to fabricating polymer-based composite with enhanced properties is strongly limited, $[17,18]$ which is mainly due to the following two factors: first, raw CNTs tend to aggregate because of van der Waals forces, consequently making CNTs very difficult to homogeneously disperse in polymer matrices; second, lack of sufficient interfacial interaction between the two phases makes CNTs easily slide in the matrices under external forces. One effective way to improve both the dispersion and interfacial bonding is to chemically functionalize CNTs $[17,19,20]$. The acid-treatment method is widely adopted to modify CNTs. In general, this process consists of two steps. First, CNTs are treated with oxidants to introduce carboxylic acid groups to CNTs' surface defect sites [21,22]. Second, the attached carboxylic acid groups could change into other functional groups, like acyl chloride, amide $[13,23]$. The functional groups on CNTs' surface can react directly with monomers or a polymer, which opens a new route to properly functionalize CNTs based on a specific polymer matrix.

Polyurethane (PU) is one of the most versatile polymers and is widely applied as thermoplastic elastomer, adhesive, coating, foam, etc. As a block copolymer with alternating soft and hard 

segments, PU's molecular structure can be easily tailored to meet specific property requirements [24]. There are several approaches to preparation of CNT/polymer composites, such as in situ polymerization, melt processing and solution mixing. Among them, solution mixing may be the most common one due to its simple procedure. Another benefit of this approach is that the de-aggregation and dispersion of CNTs can be greatly promoted by agitation, which is usually provided by ultrasonication, shear mixing, magnetic stirring or reflux [25].

In this work, in order to obtain a uniform dispersion of multi-walled carbon nanotubes (MWCNTs) in the PU matrix, as well as a strong interfacial interaction between them, two kinds of chemical moieties (carboxylic acid group and polycaprolactone diol) were introduced onto the surface of MWCNTs. Because polycaprolactone diol (PCL) is one of the monomers for synthesizing PU, we expect a good compatibility between PCL-functionalized MWCNTs (MWCNT-PCL) and PU matrix when MWCNTs also contain the same molecules on their surface. The raw MWCNTs, carboxylic acid groups functionalized MWCNTs (MWCNT-COOH) and MWCNT-PCL were then incorporated into PU matrices to prepare three types of MWCNTs/PU composites via a simple solution mixing method. The functionalized MWCNTs were characterized in detail to confirm the covalent linkage. In addition, results from FESEM, TEM, tensile tests and TGA were presented and studied.

\section{Experimental}

\subsection{Materials}

The MWCNTs (purity 95 wt\%) were provided by Cheap Tubes Inc (USA), with a length of $10-501 \mathrm{~m}$ and outer diameter of 8-15 nm. Acetone (Aik Moh), anhydrous dimethylformamide (DMF, Tritech Scientific), tetrahydrofuran (THF, Anhydrosolv, Tritech Scientific), concentrated sulfuric acid $\left(\mathrm{H}_{2} \mathrm{SO}_{4}, 95-97 \%\right.$, Honeywell) and nitric acid $\left(\mathrm{HNO}_{3}, 69-70 \%\right.$, Honeywell) were used as received. PCL (average $\mathrm{Mn} \mathrm{"'"2000} \mathrm{g/mol} \mathrm{and} \mathrm{"'"530} \mathrm{g/mol),}$ 4,4'-methylenebis(phenyl isocyanate) (MDI, molecular weight $250.25 \mathrm{~g} / \mathrm{mol}$ ), 1,4-butanediol (BD, molecular weight 90.12 $\mathrm{g} / \mathrm{mol}$ ), dibutyltin dilaurate (DBTDL) and thionyl chloride $\left(\mathrm{SOCl}_{2}\right)$ were all obtained from Sigma-Aldrich.

\subsection{Functionalization of MWCNTs}

MWCNT-COOH was obtained via the following process: raw MWCNTs $(1 \mathrm{~g})$ were dispersed in $100 \mathrm{ml}$ mixture of $\mathrm{H}_{2} \mathrm{SO}_{4} / \mathrm{HNO}_{3}$ (volumetric ratio 3:1) using an ultrasonicator. Then the mixture was heated gradually to $90 \mathrm{oC}$ and kept for $30 \mathrm{~min}$ with vigorous magnetic stirring under reflux. Afterward, the reaction system was diluted and filtered via a filter with $0.22 \mathrm{Im}$ Millipore membrane. The filtered powders were then washed, filtered till the filtrate became neutral. Finally, the acid-treated MWCNTs were collected and desiccated to a constant weight.

To prepare MWCNT-PCL, $\mathrm{SOCl}_{2}$ was used for further functionalization. The as-prepared MWCNT-COOH was dispersed in $\mathrm{SOCl}_{2}$ with the aid of an ultrasonic bath. Then the mixture was kept in a $65 \mathrm{oC}$ oil bath under reflux for 1 day. After that, the mixture was filtered via a $0.22 \mathrm{Im}$ polytetrafluoroethylene (PTFE) membrane filter. Dry acetone was used to wash the filter cake to get the acyl chloride intermediate MWCNT-COCl solid. The solid was immediately transferred into a solution of PCL (average Mn "'"530 g/mol) in dry THF and stirred at $60 \mathrm{oC}$ for another $24 \mathrm{~h}$. Afterward, the reaction mixture was filtered, washed and dried to give MWCNT-PCL. The schematic for the preparation of MWCNT-COOH and MWCNT-PCL is shown in Fig. 1.

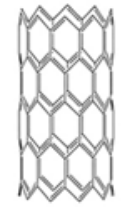

(MWCNT)

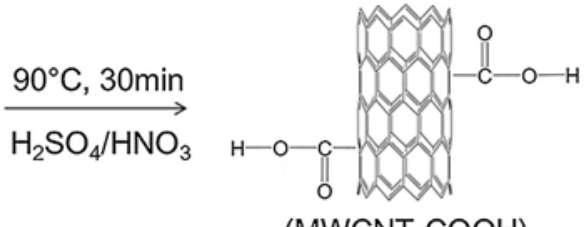

(MWCNT-COOH)

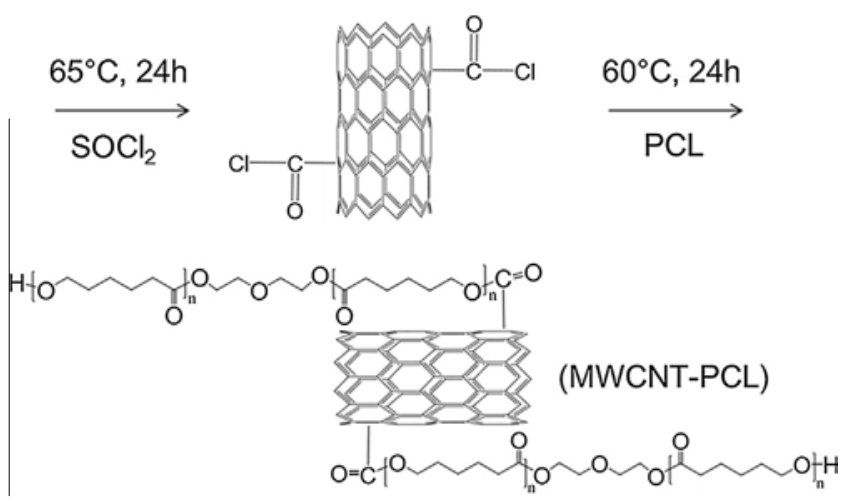

Fig. 1. Schematic for preparation of MWCNT-COOH and MWCNT-PCL.

\subsection{Fabrication of MWCNTs/PU composites}

PU was synthesized from two monomers (MDI and PCL) via a prepolymerization method [26], using BD and DBTDL as the chain extender and catalyst, respectively. A molar ratio of 1:6:5 of PCL: MDI: BD was adopted, giving $50.6 \mathrm{wt} \%$ of soft segment. The MWCNTs/PU composite films were fabricated through a solution mixing method. For the MWCNT-PCL/PU composite film, the procedure was as follows: MWCNT-PCL was firstly dispersed in DMF solution at a concentration of $0.6 \mathrm{~g} / \mathrm{L}$ and ultrasonicated at room temperature for $1.5 \mathrm{~h}$. A certain amount of as-prepared pure PU was added into the solution and dissolved with assistance of magnetic stirring. After PU was completely dissolved, the mixture was stirred continuously at room temperature for $48 \mathrm{~h}$ [27]. Then the mixture was put into an ultrasonic bath for another $1.5 \mathrm{~h}$. At last, the MWCNT-PCL/PU composite film was prepared by solution casting. The other two composite films (raw MWCNT/PU and MWCNT-COOH/PU) were prepared using the same procedure, while pure PU film was obtained without incorporating any MWCNTs. In this study, the fractions of MWCNTs for all types of MWCNTs/PU composites were fixed at $1 \mathrm{wt} \%$. The weight percent of functionalized MWCNTs in the composite was calculated based on the weight of MWCNTs before functionalization, which did not include the amount of grafted functional groups or PCL which was determined by TGA tests as shown later.

\subsection{Characterization}

FTIR spectroscopic tests were conducted on FTIR Frontier (from Perkin Elmer) with Attenuated Total Reflection (ATR). The filler samples were prepared by mixing MWCNTs with potassium bromide and pressing into transparent pellets in a pellet-forming die. The FTIR spectra of film samples were obtained using ATR. Confocal Raman spectroscopy (Witec alpha300 SR) was applied to characterize the structural changes of MWCNTs, using a $633 \mathrm{~nm}$ laser as the light source. Thermo-gravimetric analysis (TGA) measurements were used to determine the functionalization degrees of MWCNT-COOH and MWCNT-PCL, conducted on TGA Q500. The powder samples were heated from $25 \mathrm{oC}$ to $900 \mathrm{oC}$ at a speed of $10 \mathrm{oC} / \mathrm{min}$ in the presence of nitrogen. The same TGA 
instrument was also used to study the thermal stability of MWCNTs/PU composite films scanning from 25 oC to 600 oC in the presence of nitrogen. Transmission Electron Microscopy (TEM) characterization was conducted on a Carl Zeiss LIBRA ${ }^{\circledR} 120$ in-column energy filter TEM equipped with an integrated OMEGA filter. The film samples for TEM were microtomed to prepare flakes with a thickness of approximate $50-100 \mathrm{~nm}$ using Leica Ultracut UCT. Field-Emission Scanning Electron Microscopy (FESEM, JSM7600F) was used to observe the morphological features of composite films. For FESEM sample preparation, the films were broken in liquid nitrogen and sputtered with a thin layer of platinum using a sputter coater. Tensile Tester Instron 5567 was used to investigate the tensile properties of MWCNTs/PU composite films based on ASTM D638 at an extension speed of $100 \mathrm{~mm} / \mathrm{min}$. The gauge length of the specimen was $9.5 \mathrm{~mm}$ and five specimens were tested for each composite.

\section{Results and discussion}

\subsection{Characterization of functionalized MWCNTs}

The FTIR spectra of raw MWCNT, MWCNT-COOH, MWCNT-PCL and PCL are presented in Fig. 2. After acid treatment, one obvious peak at $3434 \mathrm{~cm}^{-1}$ could be observed, and it was associated with the $\mathrm{OAH}$ stretching of carboxylic acid group. Besides, the two peaks approximately at $1726 \mathrm{~cm}^{-1}$ and $1210 \mathrm{~cm}^{-1}$ could be attributed to the $\mathrm{C} @ O$ and $\mathrm{CAO}$ stretching, respectively. The result indicated that the carboxylic acid groups were successfully grafted to the surface of MWCNTs. As shown in Fig. 1, the MWCNT-COOH was further treated with $\mathrm{SOCl}_{2}$ and then reacted with PCL to prepare MWCNT-PCL. From Fig. 2, it is also clear that PCL was grafted to MWCNTs. Apart from the peaks around $3434 \mathrm{~cm}^{-1}$ (OAH stretching), $1726 \mathrm{~cm}^{-1}$ (C@O stretching) and $1210 \mathrm{~cm}^{-1}$ (CAO stretching), several new peaks appeared for the MWCNT-PCL compared with MWCNT-COOH. The peaks at around $2943 \mathrm{~cm}^{-1}$ and $2867 \mathrm{~cm}^{-1}$ were due to the asymmetrical and symmetrical stretching vibrations of the $\mathrm{CAH}$ bonds of methylene groups in the alkane chain [28]. The peaks at around $1458 \mathrm{~cm}^{-1}$ and $1089 \mathrm{~cm}^{-1}$ could be associated with the $\mathrm{CH}_{2}$ bending and CAOAC stretching modes, respectively [29].

The functionalization of MWCNTs was also evaluated with Raman tests. As shown in Fig. 3, the band at approximate $1579 \mathrm{~cm}^{-1}$ corresponded to tangential mode (G-band), which was related to the ordered $\mathrm{sp}^{2}$-hybridized carbon network. The

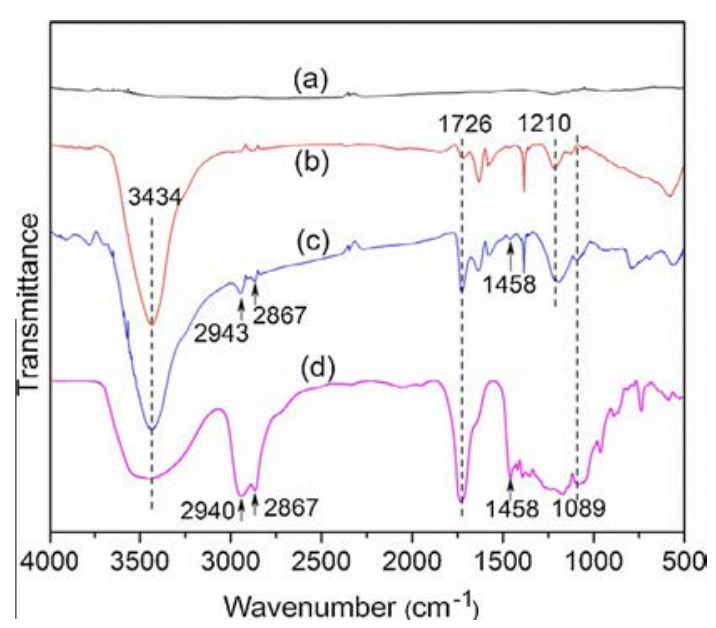

Fig. 2. FTIR spectra of raw MWCNT (a), MWCNT-COOH (b), MWCNT-PCL (c) and PCL (d). (For interpretation of the references to color in this figure legend, the reader is referred to the web version of this article.)

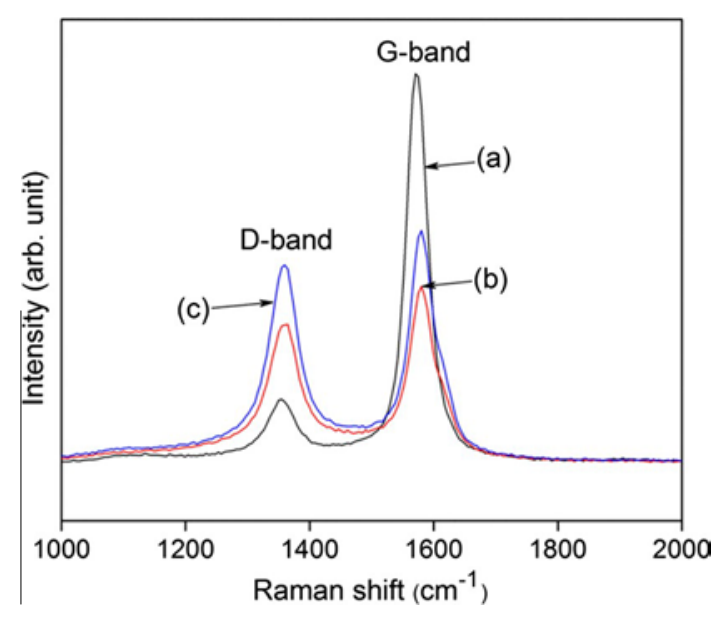

Fig. 3. Raman spectra of raw MWCNT (a), MWCNT-COOH (b) and MWCNT-PCL (c). (For interpretation of the references to color in this figure legend, the reader is referred to the web version of this article.)

band at approximate $1360 \mathrm{~cm}^{-1}$ corresponded to disorder mode (D-band), which was associated with surface defects of MWCNTs [30]. The functionalization degree of MWCNTs can be measured by the intensity ratio of the D-band and G-band $\left(\mathrm{I}_{\mathrm{D}} / \mathrm{I}_{\mathrm{G}}\right)$ [31]. It is clear that $I_{D}$ and $I_{G}$ changed significantly after functionalization. The intensity ratios $\left(\mathrm{I}_{\mathrm{D}} / \mathrm{I}_{\mathrm{G}}\right)$ of MWCNT-COOH and MWCNT-PCL were 0.96 and 0.97 , respectively, while raw MWCNT's $\mathrm{I}_{\mathrm{D}} / \mathrm{I}_{\mathrm{G}}$ was only 0.72 . This result revealed that defects or functional groups were formed on the surface of MWCNTs after chemical modification. Besides, $\mathrm{I}_{\mathrm{D}} / \mathrm{I}_{\mathrm{G}}$ of MWCNT-PCL was marginally larger than that of MWCNT-COOH, which may be associated with the electricalstructure change of MWCNT-COOH caused by $\mathrm{SOCl}_{2}$ modification [32]. In addition, compared with raw MWCNT, the D-band and G-band of the functionalized MWCNT (MWCNT-COOH and MWCNT-PCL) shifted to higher wavenumbers by 6 and $9 \mathrm{~cm}^{-1}$, respectively. This should be attributed to the covalent functionalization of organic moieties to the surface of raw MWCNT.

The results of FTIR and Raman characterizations are further supported by TEM and TGA tests. As presented in Fig. 4a, the raw MWCNT exhibited a comparatively smooth and clean surface due to its complete lattice structure of carbon network. However, the edges of MWCNT-COOH (Fig. 4b) appeared to be very rough due to the attachment of carboxylic acid groups on MWCNTs' surface. Moreover, it can be observed from Fig. 4c that a core-shell structure consisting of a PCL layer as the shell and MWCNT as the core is formed for MWCNT-PCL, indicating that the MWCNT was coated by a layer of PCL chains. It is noteworthy that the thickness of PCL layer wrapped on MWCNTs' surface is not uniform, which may be due to the inhomogeneous defect sites generated on MWCNTs' surface [33]. The TGA test under a nitrogen atmosphere also confirmed the successful functionalization of functional groups and PCL on the surface of MWCNTs. From the TGA curves of raw MWCNT, MWCNT-COOH, and MWCNT-PCL in Fig. 5, the amounts of grafted functional groups and PCL on the MWCNTs' surface were estimated to be $9.0 \mathrm{wt} \%$ and $25.3 \mathrm{wt} \%$, respectively.

\subsection{Interaction between MWCNTs and PU}

For MWCNTs/PU composite film, the interfacial bonding between MWCNTs and PU matrix was investigated with FTIR based on the shift of absorption peak of certain key group in PU matrix. Fig. 6 presents the FTIR spectra of pure PU, raw MWCNT/PU composite, MWCNT-COOH/PU composite and MWCNT-PCL/PU composite. The peak at $3332 \mathrm{~cm}^{-1}$ in Fig. 6a was attributed to 

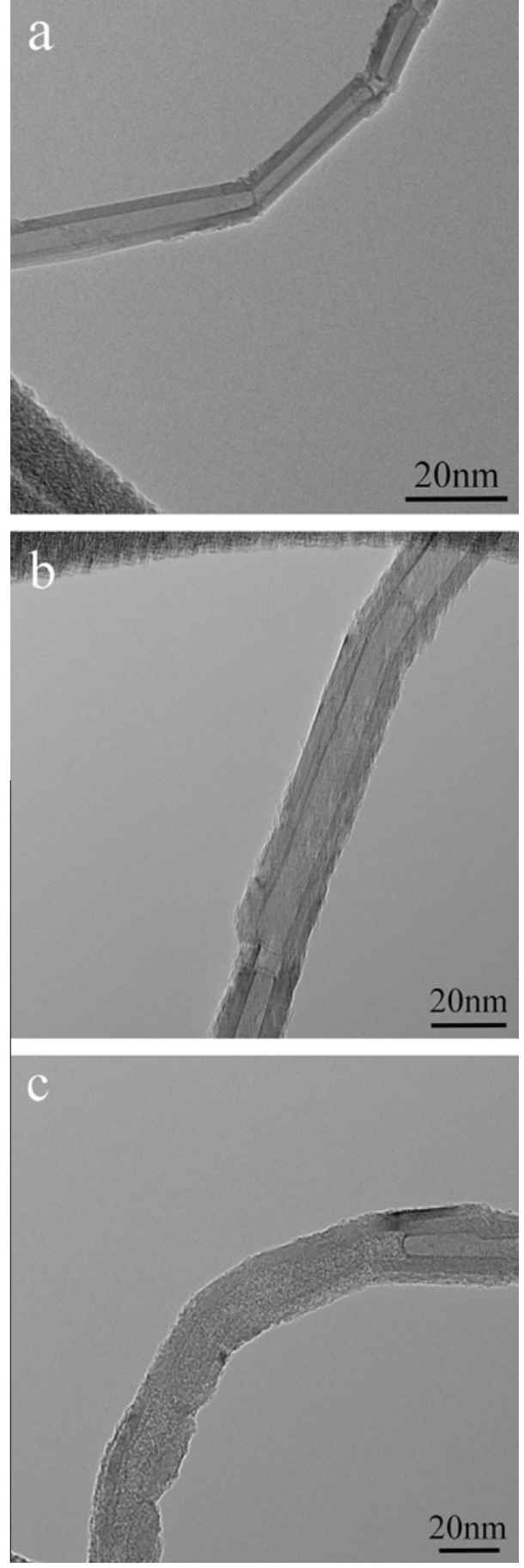

Fig. 4. TEM images of raw MWCNT (a), MWCNT-COOH (b) and MWCNT-PCL (c).

the NAH stretching vibration of the urethane linkages of PU. It can be observed that the NAH stretching peak shifted from $3332 \mathrm{~cm}^{-1}$ for pure PU and raw MWCNT/PU composite to lower wavenumbers-3326 $\mathrm{cm}^{-1}$ for MWCNT-COOH/PU composite and $3323 \mathrm{~cm}^{-1}$ for MWCNT-PCL/PU composite. This revealed that the MWCNT$\mathrm{COOH} / \mathrm{PU}$ and MWCNT-PCL/PU composites were not just simple compounds of PU and MWCNTs but contained strong chemical interaction between them, while there was almost no chemical interaction between PU and raw MWCNT.

The red shift of the NAH stretching peak suggested that the NAH groups of the urethane linkages of PU became not "free", due to the addition of MWCNT-COOH and MWCNT-PCL. In order

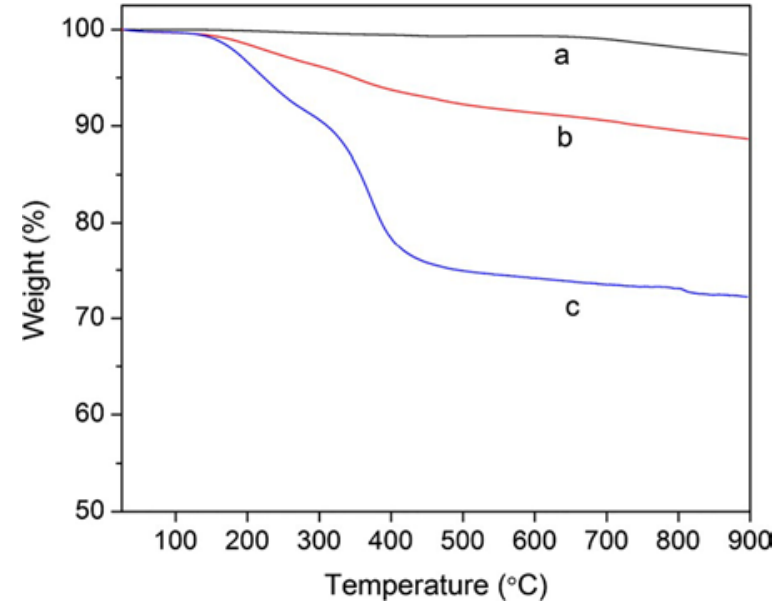

Fig. 5. TGA curves of raw MWCNT (a), MWCNT-COOH (b) and MWCNT-PCL (c) (For interpretation of the references to color in this figure legend, the reader is referred to the web version of this article.)

to explain the red shift, the hydrogen bonding between MWCNTPCL and PU matrix was proposed because the PCL chains functionalized on MWCNTs' surface were very likely to form chemical interaction with the NAH groups of PU. The possible hydrogen bonding between MWCNT-PCL and PU matrix is proposed in Fig. 7. The situation in the MWCNT-COOH/PU composite was about the same. As shown, the red shift of NAH stretching peak of MWCNT-PCL/PU composite was more prominent than that of MWCNT-COOH/PU composite. This may be because the PCL chains could provide more carbonyl groups, which were essential to the proposed hydrogen bonding, than the carboxylic acid groups. The hydrogen bonding may contribute to a better dispersion of functionalized MWCNTs in the PU matrix and more effective interfacial stress transfer between them. These suggestions are well supported by the observed dispersion of MWCNTs in the PU matrix and enhanced mechanical strength of composite films, as shown later.

\subsection{Dispersion of MWCNTs in PU}

The dispersion of MWCNTs in a polymer matrix influences the properties of MWCNT/polymer composite greatly. Hence, FESEM and TEM were used to verify the dispersion of MWCNTs in the PU matrix. Fig. 8 shows the FESEM images of the cross-sectional fractures of three studied composites: raw MWCNT/PU, MWCNT$\mathrm{COOH} / \mathrm{PU}$ and MWCNT-PCL/PU. The inserted images in Fig. 8 show the locally aggregated area under higher magnification. The bright dots in the FESEM images represent the individual MWCNTs dispersed in the PU matrix. A poor dispersion of raw MWCNT/PU composite can be clearly observed in Fig. 8a, with many large MWCNTs clusters in the matrix. Though manufactured under exactly the same sample preparation procedure, MWCNT-COOH/ PU composite (Fig. 8b) displayed a better dispersion of MWCNTs with only a few small clusters. Moreover, a homogeneous dispersion was achieved in the MWCNT-PCL/PU composite. The PU matrix was filled uniformly with MWCNT-PCL. The improved dispersion of functionalized MWCNTs in the PU matrix was also confirmed by TEM tests, as shown in Fig. 9. Since all the films were prepared by solution casting, there was no preferred orientation for all types of MWCNTs. It can be clearly seen that MWCNT-PCL were distributed more uniformly in the matrix than MWCNT$\mathrm{COOH}$, while very large aggregates of raw MWCNTs were shown in the raw MWCNT/PU composite. The results indicated that the grafted carboxylic acid groups and PCL chains could significantly 

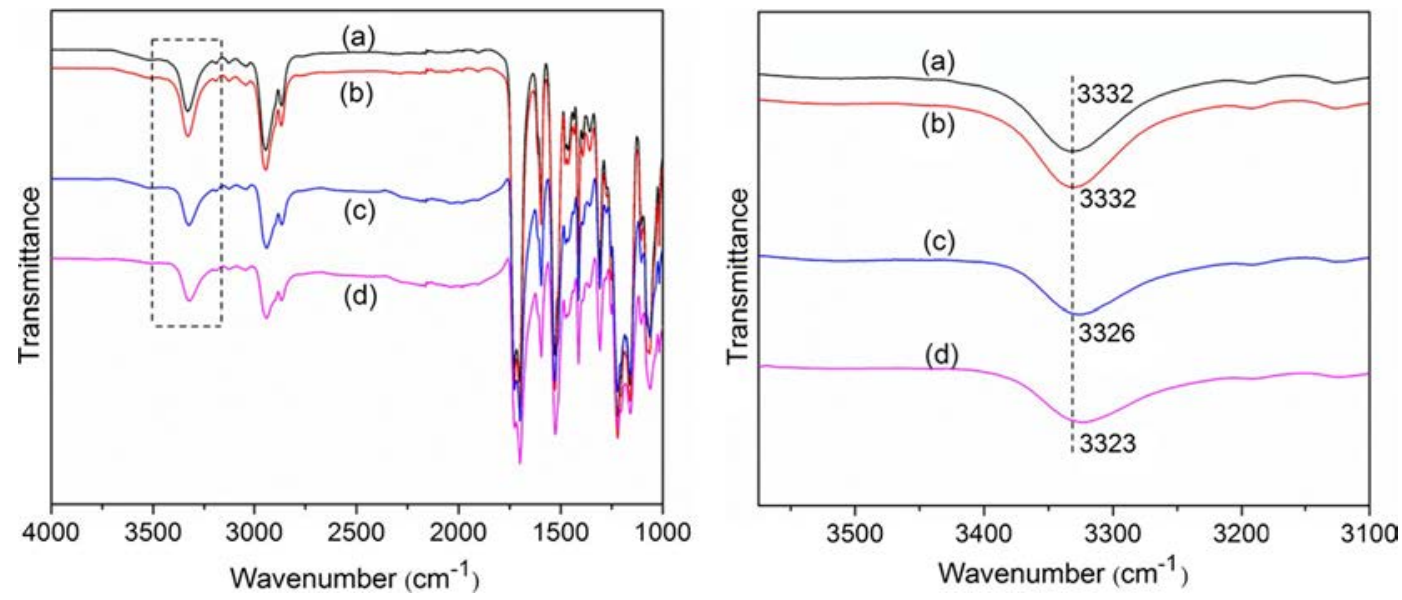

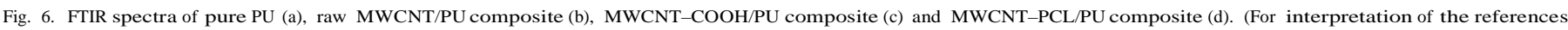
to color in this figure legend, the reader is referred to the web version of this article.)

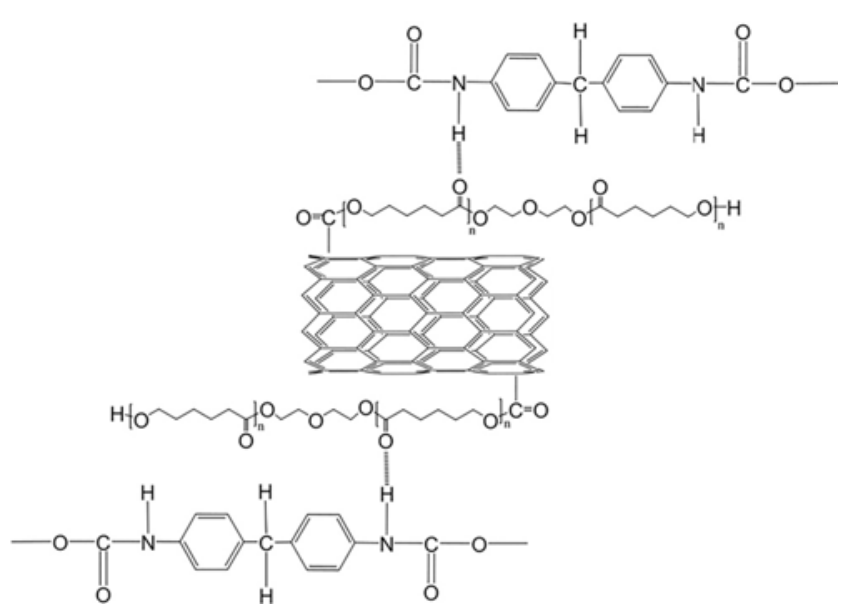

Fig. 7. Schematic of hydrogen bonding between MWCNT-PCL and PU matrix.

improve the dispersion of MWCNTs in the PU matrix. As confirmed with FTIR, the functional groups on MWCNTs' surface could form hydrogen bonding between MWCNTs and the PU matrix, which reinforced the MWCNT-PU interface and weakened the van der Waals force among the MWCNTs. Compared with MWCNT$\mathrm{COOH} / \mathrm{PU}$ composite, the better dispersion in MWCNT-PCL/PU composite may result from its stronger interfacial bonding, as discussed in the previous part.

\subsection{Mechanical performance of MWCNTs/PU composites}

Mechanical properties of MWCNTs/PU composites were investigated employing tensile tests. Fig. 10 exhibits the representative stress-strain curves for pure PU as well as for three composites under study. All the film composites showed a nonlinear elastic behavior, so the modulus was determined by taking the slope of the curve at low strains $(0-8 \%)$, while the tensile strength was determined by the maximum achieved stress. Fig. 11 presents detailed information about the tensile strength and modulus. In general, the improvement in mechanical properties of PU by the functionalized MWCNTs (MWCNT-COOH and MWCNT-PCL) was much more evident than by raw MWCNTs. The tensile strength of raw MWCNT/PU composite even decreased slightly compared to pure PU. This was very likely attributed to the poor dispersion and lack of interfacial interaction between raw MWCNTs and the
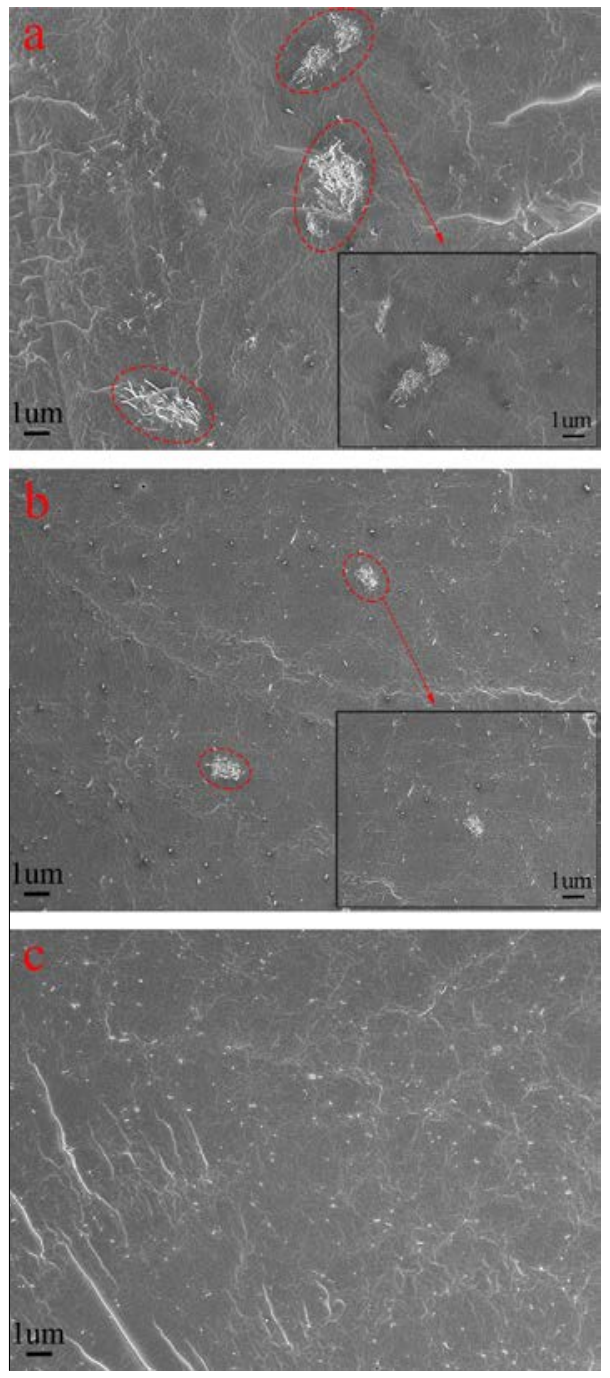

Fig. 8. FESEM images of the cross-sectional fractures of raw MWCNT/PU composite (a), MWCNT-COOH/PU composite (b) and MWCNT-PCL/PU composite (c). (For interpretation of the references to color in this figure legend, the reader is referred to the web version of this article.)

PU matrix. On the other hand, with 1 wt\% loading, the functionalized MWCNTs improved both tensile strength and modulus without sacrificing the elongation at break of pure PU. Apparently, 

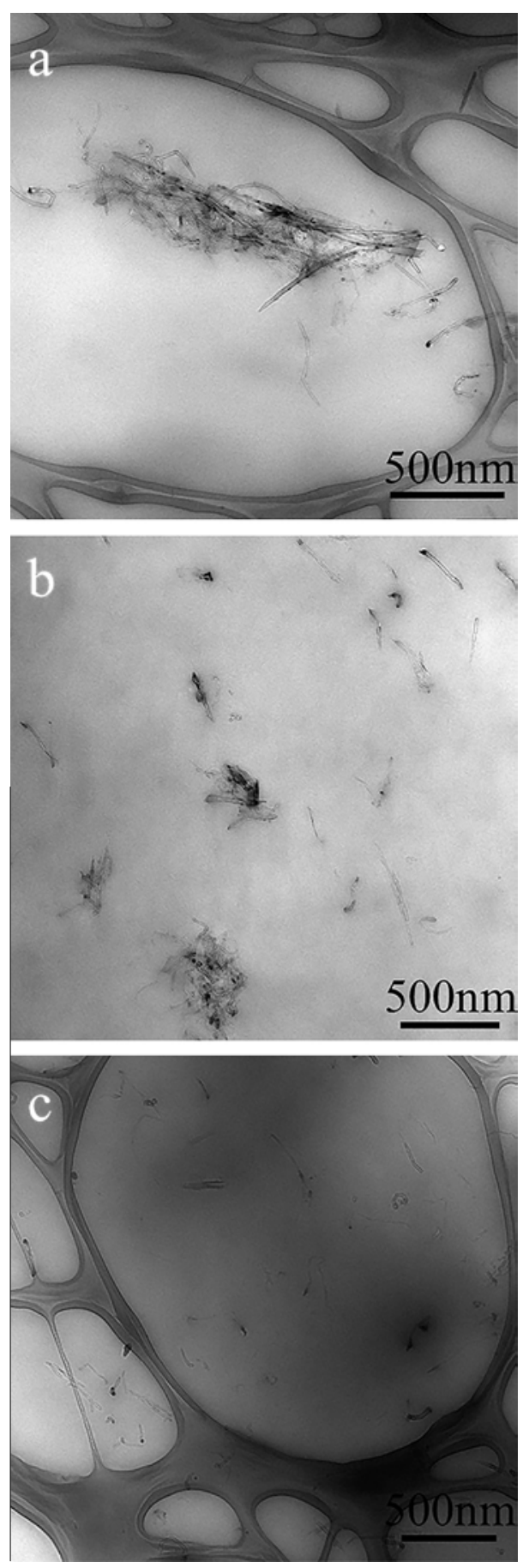

Fig. 9. TEM images of raw MWCNT/PU composite (a), MWCNT-COOH/PU composite (b) and MWCNT-PCL/PU composite (c).

the highest enhancement was achieved for the MWCNT-PCL/PU composite. Compared with pure PU, the tensile strength and modulus of the MWCNT-PCL/PU composite were $51.2 \%$ and 33.5\% higher, respectively. The FESEM images demonstrate that MWCNT-PCL was homogeneously dispersed in the PU matrix, which was crucial to improving the mechanical properties. Besides, as confirmed with FTIR, the strong interfacial interaction between MWCNT-PCL and PU matrix was beneficial for the interfacial stress transfer. Therefore, MWCNT-PCL showed a better enhancement effect than MWCNT-COOH, which may result from similarity of molecular structures of PCL and PU. With various functional groups

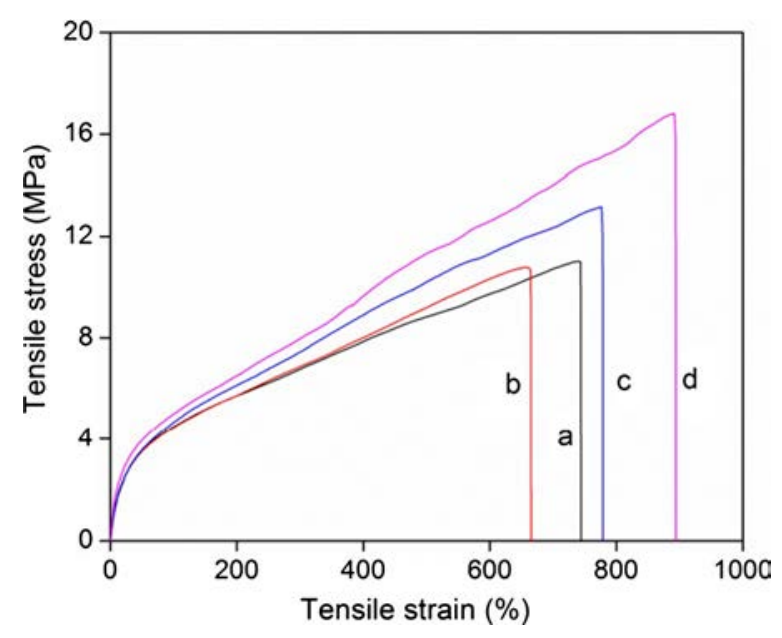

Fig. 10. Typical stress-strain curves: (a) pure PU; (b) raw MWCNT/PU composite; (c) MWCNT-COOH/PU composite; (d) MWCNT-PCL/PU composite. (For interpretation of the references to color in this figure legend, the reader is referred to the web version of this article.)
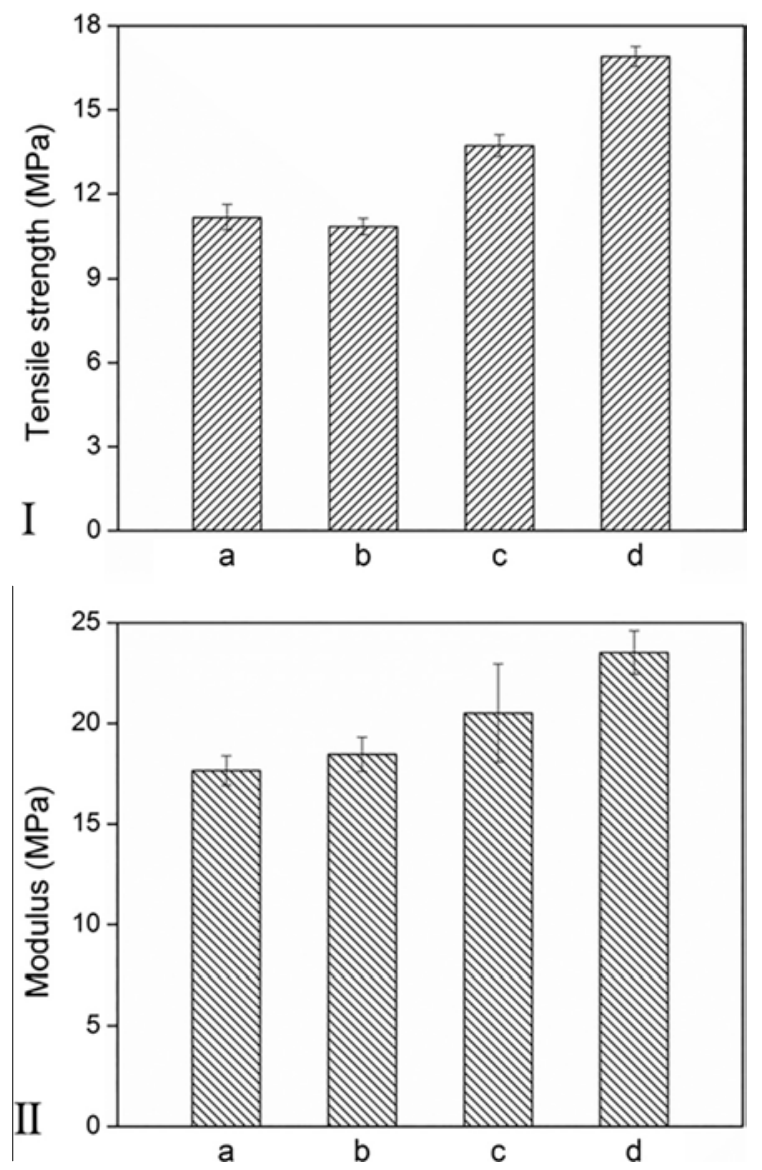

Fig. 11. Tensile strength (I) and modulus (II): (a) pure PU; (b) raw MWCNT/PU composite; (c) MWCNT-COOH/PU composite; (d) MWCNT-PCL/PU composite.

in PCL, such as ester, ether and hydroxyl, MWCNT-PCL could form stronger interaction with PU matrix than MWCNT-COOH.

\subsection{Thermal stability of MWCNTs/PU composites}

TGA thermograms of pure PU and three studied composites (Fig. 12) demonstrate that the curves shifted toward higher 


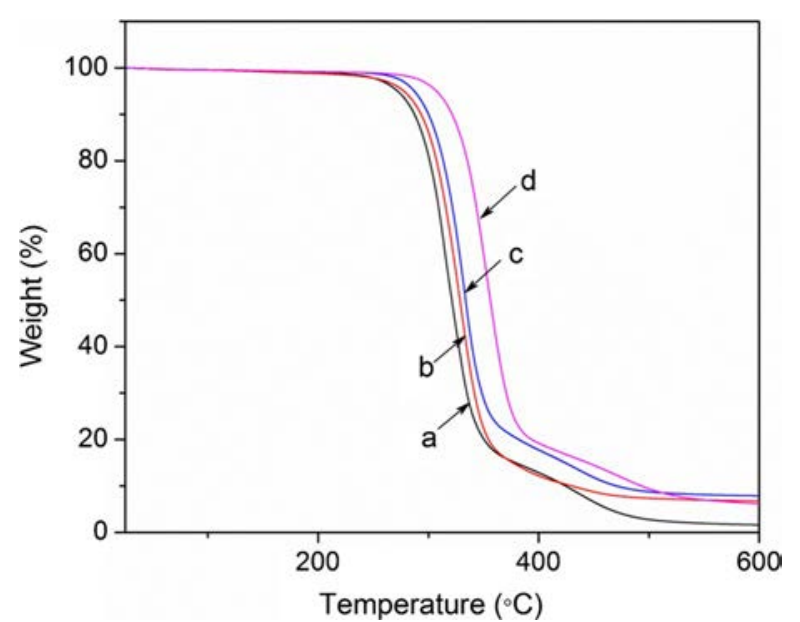

Fig. 12. TGA thermograms: (a) pure PU; (b) raw MWCNT/PU composite; (c) MWCNT-COOH/PU composite; (d) MWCNT-PCL/PU composite. (For interpretation of the references to color in this figure legend, the reader is referred to the web version of this article.)

temperature with the addition of raw MWCNT, MWCNT-COOH and MWCNT-PCL. In this work, the criteria for thermal stability were taken as the temperatures at which $2 \%\left(\mathrm{~T}_{2 \%}\right)$ and $50 \%$ $\left(\mathrm{T}_{50 \%}\right)$ weight-loss happened [13]. Compared with pure $\mathrm{PU}, \mathrm{T}_{2 \%}$ of raw MWCNT/PU composite was about the same, while $\mathrm{T}_{2 \%}$ of MWCNT-COOH/PU and MWCNT-PCL/PU composites were increased by $22 \mathrm{oC}$ and $38 \mathrm{oC}$, respectively. For $\mathrm{T}_{50 \%}$, raw MWCNT/ $\mathrm{PU}, \mathrm{MWCNT}-\mathrm{COOH} / \mathrm{PU}$ and MWCNT-PCL/PU composites showed increments of $8 \mathrm{oC}, 13 \mathrm{oC}$, and $35 \mathrm{oC}$, respectively, as compared with pure PU. Obviously, the thermal stability of PU was significantly enhanced with the addition of MWCNTs, especially MWCNT-PCL. The increased thermal stability may result from high thermal conductivity of MWCNTs, which could promote heat dissipation in the PU matrix [34]. The interfacial bonding between functionalized MWCNTs and PU matrix may further promote the heat dissipation, thus delaying the decomposition of the functionalized MWCNTs/ PU composites more effectively. In addition, the more uniform dispersion and stronger interfacial interaction in MWCNT-PCL/PU composite may contribute to its higher thermal stability compared to MWCNT-COOH/PU composite.

\section{Conclusions}

In this work, polycaprolactone diol (PCL) was one of the components to synthesize PU, and PCL was also selectively used to functionalize MWCNTs, in order to achieve a high compatibility of functionalized MWCNTs with the PU matrix. Three types of MWCNTs/PU composites (raw MWCNT/PU, MWCNT-COOH/PU and MWCNT-PCL/PU) with $1 \mathrm{wt} \%$ loading were prepared with a solution mixing method. The successful functionalization of carboxylic acid groups and PCL chains on MWCNTs' surface was confirmed with FTIR, Raman, TEM and TGA tests. A uniform dispersion of MWCNTs in the PU matrix was achieved in the MWCNT-PCL/PU composite, as verified with FESEM and TEM. Tensile tests showed that the tensile strength and modulus of MWCNT-PCL/PU composite were improved significantly (by $51.2 \%$ and $33.5 \%$, respectively) without sacrificing the elongation at break, as compared to pure PU. Regarding the thermal stability, $\mathrm{T}_{2 \%}$ and $\mathrm{T}_{50 \%}$ of MWCNT-PCL/PU composite were increased by $38 \mathrm{oC}$ and 35 oC, respectively, as compared to pure PU. Among pure PU and the three types of MWCNTs/PU composites, the highest enhancement effect in both mechanical properties and thermal stability, found for the MWCNT-PCL/PU composite, could be attributed to the homogeneous dispersion of MWCNT-PCL in the
PU matrix and strong interfacial bonding between them. Since the monomer of a polymer is generally well compatible with the polymer itself, the approach based on the use of monomer-functionalized nanofillers to improve mechanical and thermal properties of polymer materials is very promising.

\section{Acknowledgements}

The financial support from Institute for Sports Research of Nanyang Technological University (NTU) is appreciated. The FESEM and TEM work were performed at the Facility for Analysis, Characterization, Testing and Simulation (FACTS) of NTU.

\section{References}

[1] Iijima S. Helical microtubules of graphitic carbon. Nature 1991;354(6348): $56-8$.

[2] Wang J. Carbon-nanotube based electrochemical biosensors: a review. Electroanalysis 2005;17(1):7-14.

[3] Balasubramanian K, Burghard M. Biosensors based on carbon nanotubes. Anal Bioanal Chem 2006;385(3):452-68.

[4] Ajayan PM, Iijima S. Capillarity-induced filling of carbon nanotubes. Nature 1993;361(6410):333-4.

[5] Esplandiu MJ, Bittner VG, Giapis KP, Collier CP. Nanoelectrode scanning probes from fluorocarbon-coated single-walled carbon nanotubes. Nano Lett 2004;4(10):1873-9.

[6] Baughman RH, Cui CX, Zakhidov AA, Iqbal Z, Barisci JN, Spinks GM, et al. Carbon nanotube actuators. Science 1999;284(5418):1340-4.

[7] Baughman RH, Zakhidov AA, de Heer WA. Carbon nanotubes - the route toward applications. Science 2002;297(5582):787-92.

[8] Andrews R, Weisenberger MC. Carbon nanotube polymer composites. Curr Opin Solid St M 2004;8(1):31-7.

[9] Zou YJ, Xiang CL, Yang LN, Sun LX, Xu F, Cao Z. A mediatorless microbial fuel cell using polypyrrole coated carbon nanotubes composite as anode material. Int J Hydrog Energy 2008;33(18):4856-62.

[10] Zhang XF, Liu T, Sreekumar TV, Kumar S, Moore VC, Hauge RH, et al. Poly(vinyl alcohol)/SWNT composite film. Nano Lett 2003;3(9):1285-8.

[11] Qian D, Dickey EC, Andrews R, Rantell T. Load transfer and deformation mechanisms in carbon nanotube-polystyrene composites. Appl Phys Lett 2000;76(20):2868-70

[12] Clayton LM, Sikder AK, Kumar A, Cinke M, Meyyappan M, Gerasimov TG, et al. Transparent poly(methyl methacrylate)/single-walled carbon nanotube (PMMA/SWNT) composite films with increased dielectric constants. Adv Funct Mater 2005;15(1):101-6.

[13] Sahoo NG, Cheng HKF, Li L, Chan SH, Judeh Z, Zhao JH. Specific functionalization of carbon nanotubes for advanced polymer nanocomposites. Adv Funct Mater 2009;19(24):3962-71.

[14] Popov VN. Carbon nanotubes: properties and application. Mater Sci Eng R-Rep 2004;43(3):61-102.

[15] Sun YP, Fu KF, Lin Y, Huang WJ. Functionalized carbon nanotubes: properties and applications. Acc Chem Res 2002;35(12):1096-104.

[16] Terrones M. Science and technology of the twenty-first century: synthesis, properties and applications of carbon nanotubes. Annu Rev Mater Res 2003;33:419-501.

[17] Sahoo NG, Rana S, Cho JW, Li L, Chan SH. Polymer nanocomposites based on functionalized carbon nanotubes. Prog Polym Sci 2010;35(7):837-67.

[18] Oberdisse J, Hine P, Pyckhout-Hintzen W. Structure of interacting aggregates of silica nanoparticles in a polymer matrix: small-angle scattering and reverse Monte Carlo simulations. Soft Matter 2007;3(4):476-85.

[19] Garg A, Sinnott SB. Effect of chemical functionalization on the mechanical properties of carbon nanotubes. Chem Phys Lett 1998;295(4):273-8.

[20] Ramanathan T, Fisher FT, Ruoff RS, Brinson LC. Amino-functionalized carbon nanotubes for binding to polymers and biological systems. Chem Mater 2005;17(6):1290-5.

[21] Jung YC, Sahoo NG, Cho JW. Polymeric nanocomposites of polyurethane block copolymers and functionalized multi-walled carbon nanotubes as crosslinkers. Macromol Rapid Commun 2006;27(2):126-31.

[22] Xiong JW, Zheng Z, Song WH, Zhou DS, Wang XL. Microstructure and properties of polyurethane nanocomposites reinforced with methylenebis-ortho-chloroanilline-grafted multi-walled carbon nanotubes. Compos Part A-Appl S 2008;39(5):904-10.

[23] Xiong JW, Zheng Z, Qin XM, Li M, Li HQ, Wang XL. The thermal and mechanical properties of a polyurethane/multi-walled carbon nanotube composite. Carbon 2006;44(13):2701-7.

[24] Šebenik U, Krajnc M. Influence of the soft segment length and content on the synthesis and properties of isocyanate-terminated urethane prepolymers. Int J Adhes Adhes 2007;27(7):527-35.

[25] Coleman JN, Khan U, Blau WJ, Gun'ko YK. Small but strong: a review of the mechanical properties of carbon nanotube-polymer composites. Carbon 2006;44(9):1624-52. 
[26] Cho JW, Kim JW, Jung YC, Goo NS. Electroactive shape-memory polyurethane composites incorporating carbon nanotubes. Macromol Rapid Commun 2005;26(5):412-6.

[27] Sahoo NG, Jung YC, Yoo HJ, Cho JW. Effect of functionalized carbon nanotubes on molecular interaction and properties of polyurethane composites. Macromol Chem Phys 2006;207(19):1773-80.

[28] Yun S, Im H, Kim J. The effect of different hard segments in polyurethane on the electrical conductivity of polyurethane grafted multi-walled carbon nanotube/polyurethane nanocomposites. Synt Met 2011;161(13-14):1361-7.

[29] Silverstein Robert M, Webster Francis X, Kiemle David J. Spectrometric identification of organic compounds. Hoboken, NJ: John Wiley \& Sons; 2005. p. $72-108$.

[30] Chen XH, Wang HF, Zhong WB, Feng T, Yang XG, Chen JH. A scalable route to highly functionalized multi-walled carbon nanotubes on a large scale. Macromol Chem Phys 2008;209(8):846-53.
[31] Price BK, Hudson JL, Tour JM. Green chemical functionalization of singlewalled carbon nanotubes in ionic liquids. J Am Chem Soc 2005;127(42): $14867-70$.

[32] Dettlaff-Weglikowska U, Skakalova V, Graupner R, Jhang SH, Kim BH, Lee HJ, et al. Effect of $\mathrm{SOCl}_{2}$ treatment on electrical and mechanical properties of single-wall carbon nanotube networks. J Am Chem Soc 2005;127(14): 5125-31.

[33] Yang YK, Xie XL, Wu JG, Yang ZF, Wang XT, Mai YW. Multiwalled carbon nanotubes functionalized by hyperbranched poly(urea-urethane)s by a onepot polycondensation. Macromol Rapid Commun 2006;27(19):1695-701.

[34] Huxtable ST, Cahill DG, Shenogin S, Xue LP, Ozisik R, Barone P, et al. Interfacial heat flow in carbon nanotube suspensions. Nat Mater 2003;2(11):731-4. 